\title{
A Full Error Dynamics Switching Modeling and Control Scheme for an Articulated Vehicle
}

\author{
Thaker Nayl, George Nikolakopoulos and Thomas Gustafsson
}

\begin{abstract}
In this article, a complete analysis towards the development of a switching modeling and control framework for an articulated vehicle, under the effect of varying slip angles will be presented. The established nonlinear kinematic model, of the nonholonomic articulated vehicle, will be transformed into an error dynamics model, which in the sequel will be linearized around multiple nominal slip angle cases. The proposed control architecture will consist of a switching control scheme, based on multiple model predictive controllers, for the articulated vehicle under varying slip angles. The controllers will be developed in order to improve the performance of the articulated vehicle's path tracking, while compensating the varying slippage effect. The current measured slip angle is being considered as the switching rule and a corresponding switching control scheme is being defined, being able to apply constraints on the states, the control signal and the output variables. Both the non-slip and slip models will be derived to highlight the significance of accounting for slips in path following control and their significant effect on deteriorating the performance of the overall control scheme when not considered. Multiple simulation results will be presented to prove the efficacy of the overall suggested scheme.
\end{abstract}

Keywords: Articulated vehicle, kinematic model, model predictive control, slip angle.

\section{INTRODUCTION}

Articulated vehicles, such as the Load Haul Dump (LHD) vehicle, consist of two parts, a front and a rear, linked with a rigid free joint, while the steering action is performed on the joint, by changing the corresponding articulated angle, between the front and the rear parts of the vehicle.

During the operation of the articulated vehicle, there are additional external factors that degrade the overall system performance and introduce errors in the model that could be propagated with the time. Such deteriorating factors are: a) the generic interaction between the vehicle and its surrounding environment [1], b) the noise and bias in the positioning and driving sensors [2], c) the dynamic effects resulting from acceleration and braking [3], and d) the existence of variation of slip angles among the trailer and the tractor, which are also being influenced by the type of the driving ground, the fatigue and the tyre's type [4].

In the related literature there have been several research approaches for the problem of modeling articulated vehicles, based on the theory of multiple body dynamics such as those in [5,6]. Moreover, a mostly utilized kinematic model for the LHD vehicle, formulated from general geometry, that includes ideal and slip behaviour has been initially proposed in [7]. Most of these methods have been

\footnotetext{
Manuscript received xxxxx xx, 2014.

Thaker Nayl, George Nikolakopoulos and Thomas Gustafsson are with Luleå University of Technology, Department of Computer, Electrical and Space Engineering, Control Engineering Group, Luleå SE-971 87 Sweden.

Corresponding Author's e-mail:thaker.nayl@ltu.se
}

derived based on simple models that were not taking under consideration the effects of varying slip angles, or were considering them as static, as in the later case, the complexity of the overall problem is being increased and it is more difficult to proceed to the next stage of the control scheme design based on highly nonlinear articulated vehicle's dynamics. Finally, in $[8,9]$ it has been proposed an approximating off-axle kinematics by an on-axle system with a similar steady response, in which model differences are regarded as perturbations for the path tracker, while classical fundamental problems of motion control for articulated vehicles have been presented. Many researchers have proposed a variety of articles, focusing only on the measurement, modeling and estimation of slip angles and the way that the external environment and dynamic models are affecting them $[10,11]$, or examining the overall problem of kinematic modeling of a multi-body vehicle (multiple trailers), with articulated joints [12].

From a control point of view, there have been proposed many traditional techniques for nonholonomic vehicles, based on error dynamics models without the presence of slip angles. A control scheme that combines a kinematic controller and a sliding mode for wheeled mobile robots has been presented in [13, 14]. In [15] a general kinematic model of an articulated vehicle has been proposed that described how heading angle evolved with time as a function of steering angle and velocity. In [16] a Lyapunov based approach has been presented, that addressed the problem of asymptotic stabilization for backward motion. A nonlinear control law based on partial state feed- 
back linearization and a Lyapunov method for the closedloop path following problem of a nonholonomic mobile robot has been appeared in $[17,18]$. Finally, a control scheme based on linear matrix inequalities has been presented in [19] and in [20] a pole placement technique has been applied.

The main contributions of this article are the following ones. Firstly, a complete vehicle kinematic modeling framework for an articulated vehicle and in presence of slip angles is established. In this framework, an error dynamics modeling procedure will be derived for the case of an articulated vehicle operating under varying slip angles. Until now and mainly due to the increasing complexity of the problem, only the case of non-slip angles affecting the movement of the vehicle has been considered. In the proposed modeling framework, a linearised error modeling framework, being able to take under consideration the effect of the varying slip angles, in order to improve the overall closed loop performance is being presented.

The second contribution is related to the problem of controlling the articulated vehicle, under the presence of varying slip angles. This problem is being addressed by proposing a switching Model Predictive Control (MPC) scheme for articulated vehicles. The main advantages of the MPC, when compared with the alternative established schemes, is the ability to handle constraints on the states, the physical limitations (e.g. articulated angle) and the control signals in a straightforward way and in full symphony with the real life operating articulated vehicles. As it will be analysed, in the presented approach, multiple MPC controllers are being fine tuned for specific slippage operating conditions, while the current estimated slip angle of the front part is considered as the mode selector for the switching MPC. The resulting control scheme provides the optimal control for each region of slip angles, while ensuring smooth transition of the control effort as the articulated vehicle is driven over regions of different slippage.

Thirdly, the presented slippage modeling, combined with the MPC scheme, is being compared to existing models that are not taking under consideration the effect of the slippage, and an overall analysis and proof of the proposed performance improvement are provided through extended simulated real life test cases and the related discussions.

Finally, the fourth contribution concerns the evaluation and comparison of the proposed scheme with classical and most commonly utilized control schemes in the area of articulated vehicles, such as the Linear Quadratic Regulator (LQR) and the Pole Placement control approaches (PP).

At this point is should be highlighted that this research work will not focus on how we can model the mechanics behind the generation of the slip angles or how the dynamic modeling of the vehicle will effect the slippage. It is also further assumed that the slip angles can be either directly measured (e.g. by an external motion tracking sys- tem or by a differential GPS) or can be directly estimated. In general, the slip angles are extremely difficult to model accurately, as they are generated by the vehicle dynamics [7], while their value is fully dependable to vehicle's velocity, mass, tyre-terrain interaction and articulation angle in a highly nonlinear way.

The rest of the article is organized as follows: In Section 2, a non-slip kinematic model that rejects all the elements that give rise to slippage is being presented, and secondly a kinematic model that takes into account lateral wheel slippage that may be encountered in practice is being developed and analysed in detail. In Section 3, the switching model predictive controller is being analyzed, while in Section 4, multiple simulation results of the articulated vehicle, under the effect of various slip angles are being depicted that prove the efficacy of the proposed scheme. Finally, the concluding remarks are provided in Section 5 .

\section{ARTICULATED VEHICLE MODELING}

For deriving the various kinematic models of the articulated vehicle, it has been assumed that each body has a single axle. The vehicle's wheels are all non-steerable. The steering action is performed on the joint, by changing the corresponding articulated angle, between the front and rear parts of the vehicle, while the articulated vehicle's geometry is being indicated in Fig.1. The articulated vehicle

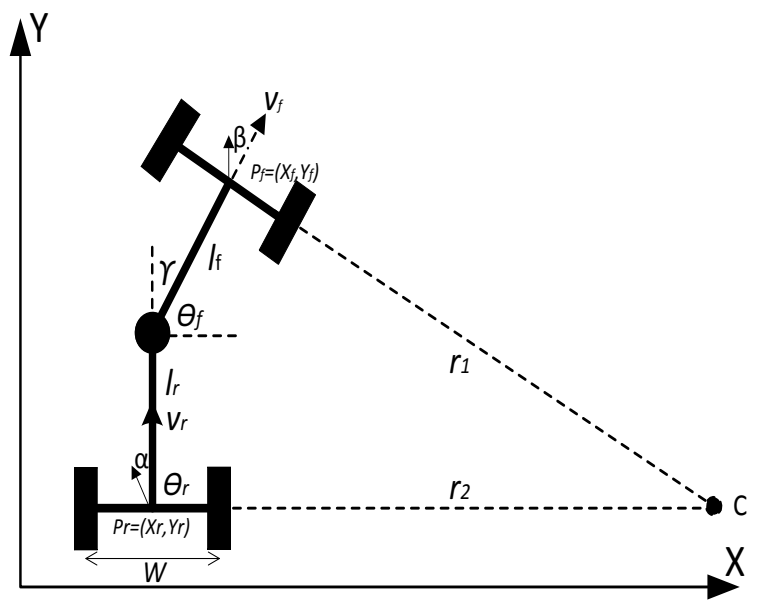

Fig 1: The articulated vehicle model.

consists of two parts, with a width of $w$ and the front and rear part has lengths $\left(l_{f}, l_{r}\right)$ correspondingly and are linked with a rigid free joint $h$, while each part has a single axle and all wheels are non-steerable. The centers of the two parts are being denoted as $P_{f}=\left(x_{f}, y_{f}\right)$ and $P_{r}=\left(x_{r}, y_{r}\right)$ respectively. The steering action is being performed on the joint, by changing the corresponding articulated angle $\gamma$, in the middle of the vehicle, while $\dot{\gamma}$ is the rate of change for the articulated steering angle. 
The velocities $v_{f}$ and $v_{r}$ are considered to have the same changing with respect to the velocity of the rigid free joint of the vehicle, $\left(\dot{\theta}_{f}, \dot{\theta}_{r}\right)$ are the angular velocities of the front and rear parts of the vehicle respectively. The definition of this model has been initially proposed based on the derivation in [21]. This model has included the perturbed factors in the vehicle position as two slip variables $\beta$ and $\alpha$, which are the front and rear slipping angles of the vehicle and also the angles between the linear velocities of the vehicle respectively.

Moreover, it is assumed that the steering angle $\gamma$ remains constant under small displacement, the dynamical effects due to low velocity, like tire characteristic, friction, load and breaking force are being neglected, each axle is composed of two wheels and each pair is being replaced by a unique wheel. In what it follows the kinematic models for the articulated vehicle under study without and with the presence of slippage will be derived.

Based on the assumption that the vehicle develops a steady-state motion turning, or $\dot{\gamma}=0$, the rates of the orientation change are being provided by: $r_{1}=\frac{v_{f}}{\dot{\theta}_{f}}, r_{2}=\frac{v_{r}}{\dot{\theta}_{r}}$, $\dot{\theta}_{f}=\frac{v_{f} \sin \gamma}{l_{f} \cos \gamma+l_{r}}+\frac{\dot{\gamma} l_{r}}{l_{f} \cos \gamma+l_{r}}$, and $\dot{\theta}_{r}=\dot{\theta}_{f}-\dot{\gamma}$.

In Fig. 2 it is depicted an overview of how the displacement, heading and curvature errors are being defined, between the actual path of the articulated vehicle and the desired one, while for this representation, an error dynamics state vector as $\dot{x}_{e}=\left[\begin{array}{lll}\dot{e}_{c} & \dot{e}_{h} & \dot{e}_{d}\end{array}\right]^{T}$, is being defined. Moreover, the distance from the vehicle to the reference path displacement, and the angle between the vehicle and the reference are also being displayed in Fig. 2 .

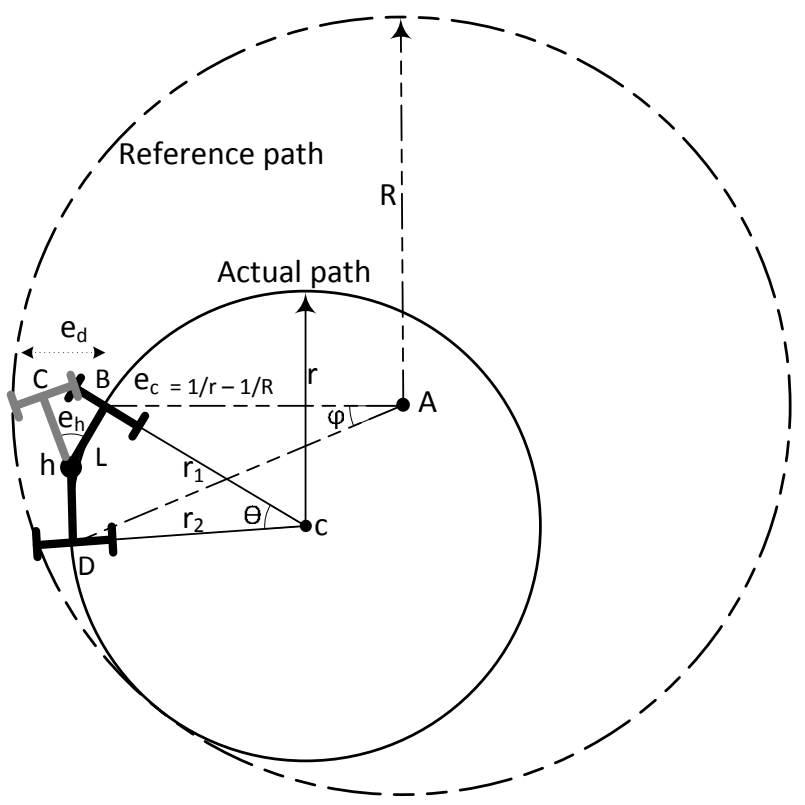

Fig 2: Path Following kinematic error dynamics model for the articulated vehicle.

In the following derivation, three errors are defined as: a) $e_{d}$ is the displacement error, b) $e_{h}$ is the heading error, and c) $e_{c}$ is the curvature error. The main assumptions to derive the error dynamic model of the articulated vehicle are that the steering angle $\gamma$ remains constant under small displacement and dynamical slipping effects, while it is assumed that the vehicle lengths are equal, $L=l_{f}=l_{r}$.

The displacement error $e_{d}$ is the difference between the coordinates of the tractor and the coordinates of the desired circular path. From the triangle (ABD), it can be defined that $\theta=\arctan \frac{L}{r}$. From the triangle (CBD), it is assumed that $\phi$ and $\theta$ are small, can get the relation of,

$$
e_{d}=L . e_{h}
$$

Furthermore, the vehicle length $L$ can be defined as $L=$ $r \theta$ and the displacement error, $e_{d}=r \theta e_{h}$, afterwards, taking the time derivative of this equation yields:

$$
\dot{e}_{d}=v e_{h}
$$

The heading error $e_{h}$ is the orientation difference between the centers of the vehicle and the circular path. A change in the heading error is being defined as $e_{h}=\theta-\phi$, while the relation between $\theta$ and $\phi$ with respect to $L$, after small assumptions, is defined as: $(\theta r=\phi R)$. From this equation it can be derived that,

$$
e_{h}=\frac{\theta R-\theta r}{R}
$$

and by taking the time derivative results in:

$$
\dot{e}_{h}=\dot{\theta} r\left(\frac{1}{r}-\frac{1}{R}\right)
$$

The curvature error $e_{c}$ measures the difference between the vehicle's path circle and the curvature of the trajectory path, and it can be defined as:

$$
e_{c}=\left(\frac{1}{r}-\frac{1}{R}\right)
$$

By utilizing the relations in above equations, the curvature error is being defined as:

$$
\dot{e}_{h}=v e_{c}
$$

With the assumption that the velocity and the curvature of the trajectory are constant and by differentiating the curvature equation $e_{c}=\left(\frac{1}{r}-\frac{1}{R}\right)$ with respect to time, the rate of the curvature error is being defined as:

$$
\begin{aligned}
\dot{e}_{c}= & \frac{\dot{\gamma} l_{r} \cos (\gamma+\beta-\alpha) \cos \alpha+\dot{\gamma} l_{f} \cos (\gamma+\beta-\alpha) \cos (\gamma-\alpha)}{\left(l_{r} \cos \alpha+l_{f} \cos (\gamma-\alpha)\right)^{2}}+ \\
& \frac{\left.\dot{\gamma} v l_{f} \sin (\gamma+\beta-\alpha) \sin (\gamma-\alpha)-\dot{\gamma}^{2} l_{f} l_{r} \cos \alpha\right) \sin (\gamma-\alpha)}{v\left(l_{r} \cos \alpha+l_{f} \cos (\gamma-\alpha)\right)^{2}}- \\
& \frac{\ddot{\gamma} l_{f} l_{r} \cos (\gamma-\alpha) \sin (\alpha) \dot{\alpha}+\left(l_{r}^{2} \cos \alpha \sin \alpha \dot{\alpha}\right)}{v\left(l_{r} \cos \alpha+l_{f} \cos (\gamma-\alpha)\right)^{2}}
\end{aligned}
$$

Linearizing the error dynamics in above equation around the reference path $\gamma$ yields:

$$
\dot{e}_{c}=\dot{\gamma} \frac{\left(l_{f}+l_{r}\right)+l_{f}\left(\gamma^{2}+\gamma \beta-2 \gamma \alpha\right)+l_{f}\left(\alpha^{2}-\alpha \beta\right)}{\left(l_{r}+l_{f}\right)^{2}}
$$


where it can be observed that $\dot{e}_{c}$ is dependant on the slippage and the rate of change of articulation angles. By performing the following change of variable:

$$
\dot{e}_{c}=\dot{e}_{c}-\dot{\gamma} \frac{l_{f}\left(\gamma^{2}+\gamma \beta-2 \gamma \alpha\right)}{\left(l_{r}+l_{f}\right)^{2}}
$$

The following state space error dynamics description for the articulated vehicle is being extracted:

$$
\left[\begin{array}{c}
\dot{e}_{d} \\
\dot{e}_{h} \\
\dot{e}_{c}
\end{array}\right]=\left[\begin{array}{lll}
0 & v & 0 \\
0 & 0 & v \\
0 & 0 & 0
\end{array}\right]\left[\begin{array}{c}
e_{d} \\
e_{h} \\
\dot{e}_{c}
\end{array}\right]+\left[\begin{array}{c}
0 \\
\frac{l_{f}(1+\beta-2 \alpha)}{\left(l_{f}+l_{r}\right)^{2}} \\
\frac{l_{r}+l_{f}\left(1+\alpha^{2}-\alpha \beta\right)}{\left(l_{f}+l_{r}\right)^{2}}
\end{array}\right] \dot{\gamma}
$$

In the case that the articulated vehicle is being driven over terrains that are characterized by different slip angles, multiple larger operating sets of slip angles, with the same characteristics, $\alpha_{i} \in \mathscr{L}_{1}$ and $\beta_{i} \in \mathscr{L}_{2}$, with $\mathscr{L}_{1}, \mathscr{L}_{2} \in \mathfrak{R}^{2}$ and $i \in \mathbf{Z}^{+}$, can be defined, leading to a switching state space description for the model in (20) as it follows:

$$
\dot{\mathbf{x}}=\mathbf{A x}+\mathbf{B}_{i} \dot{\gamma}
$$

where:

$\mathbf{x}=\left[\begin{array}{c}e_{d} \\ e_{h} \\ e_{c}\end{array}\right], \mathbf{A}=\left[\begin{array}{lll}0 & v & 0 \\ 0 & 0 & v \\ 0 & 0 & 0\end{array}\right], \quad \mathbf{B}_{i}=\left[\begin{array}{c}0 \\ \frac{l_{f}\left(1+\beta_{i}-2 \alpha_{i}\right)}{\left(l_{f}+l_{r}\right)^{2}} \\ \frac{l_{r}+l_{f}\left(1+\alpha_{i}^{2}-\alpha_{i} \beta_{i}\right)}{\left(l_{f}+l_{r}\right)^{2}}\end{array}\right]$

and $\mathbf{x} \in \mathscr{X} \subseteq \mathfrak{R}^{3}$ is the state vector, $\dot{\gamma} \in \mathscr{U} \in \mathfrak{R}$ is the control action, $\mathbf{A} \in \mathfrak{R}^{3 \times 3}, \mathbf{B}_{\mathbf{i}} \in \mathfrak{R}^{3 \times 1}$, and full state feedback is being considered, or $\mathbf{C}=\mathbf{I}_{3 \times 3}$.

If the effect of the slip angles is being neglected, or equivalently $\beta_{i}=\alpha_{i}=0$, the previous $\mathbf{A}$ matrix remains unchanged, while the $\mathbf{B}_{\mathbf{i}}$ matrix is significantly simplified into the constant matrix $\mathbf{B}_{\mathbf{n s}}$, defined as:

$$
\mathbf{B}_{n s}=\left[\begin{array}{c}
0 \\
\frac{l_{f}}{\left(l_{f}+l_{r}\right)^{2}} \\
\frac{l_{r}+l_{f}}{\left(l_{f}+l_{r}\right)^{2}}
\end{array}\right]
$$

The effect of considering the case of varying slip angles on the behaviour of the vehicle and the overall change of the performance is depicted in Fig.3 during an overturned $S$-path manoeuvre in open loop. From the obtained results, it is more than obvious that the slipping conditions can dramatically deteriorate the performance of the articulated vehicle and thus a proper control scheme should be designed being able to present robustness and performance attenuation under the case of varying slip angles.

\section{MODEL PREDICTIVE CONTROL DESIGN}

Model predictive control is an optimal control strategy that uses the model of the system to obtain an optimal control sequence of future control actions. At each sampling

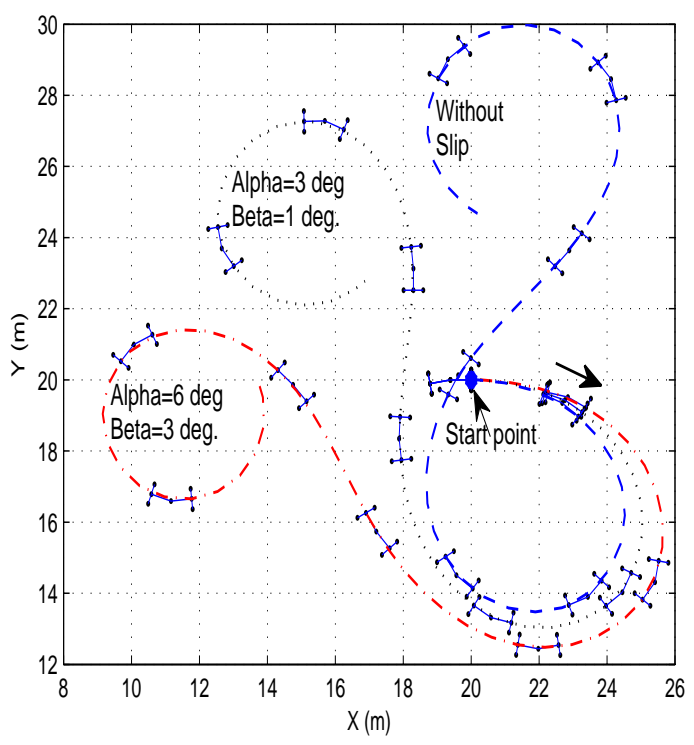

Fig 3: Open Loop Comparison of slippage effect on the articulated vehicle movements under the same commanded $S$-path.

time, the model predictive controller generates an optimal control sequence by solving an optimization problem. The first element of this sequence is applied to the plant, while the problem is solved again at the next sampling time using the updated process measurements and a shifted horizon.

Based on these predictions, an objective function is minimized with respect to the future sequence of inputs, thus requiring the solution of a constrained optimization problem for each sampling interval. Although prediction and optimization are performed over a future horizon, only the values of the inputs for the current sampling interval are used and the same procedure is repeated at the next sampling time. This mechanism is known as moving or receding horizon strategy, in reference to the way in which the time window shifts forward from one sampling time to the next one [22].

$\mathrm{MP}-$ controller has been very successful in practice and its a highly effective control scheme that is able to take under consideration multiplicative system model descriptions, uncertainties, nonlinearities and physical and mechanical constraints in the system model parameters or in the control signals. In the current research effort, the MPcontroller schemes are able to predict future values of the vehicles error dynamics based on the present available information and the current constraints [23].

The overall block diagram of the proposed switching closed loop system is depicted in Fig.4. As it analysed, multiple MPC controllers are being fine tuned for specific slipping operating conditions, while the current front slip angle is considered as the mode selector for the switching 
MPC. The parameters for the MP-controllers, for the state and control action weighings matrices, have been tuned according to the different encountered slip angles. Moreover, the prediction horizon, the control horizon, the states weight matrix, and the control weight matrix are tuned according to the different assumed cases for the varying slip angles.

For efficiently controlling the articulated vehicle, the controller utilizes the current state of motion of the vehicle as well as the next target points of the reference trajectory. In the sequel this path (planar coordinates) is being translated to curvature, heading and displacement coordinates that act as the reference input for the controller. The formulation of the switching MP-controller is based on: a) the current full state feedback, c) the active constrains on the system, d) the current values of the slip angles that act as the mode selector, while it is assumed that the vehicle is cruising under a constant speed.

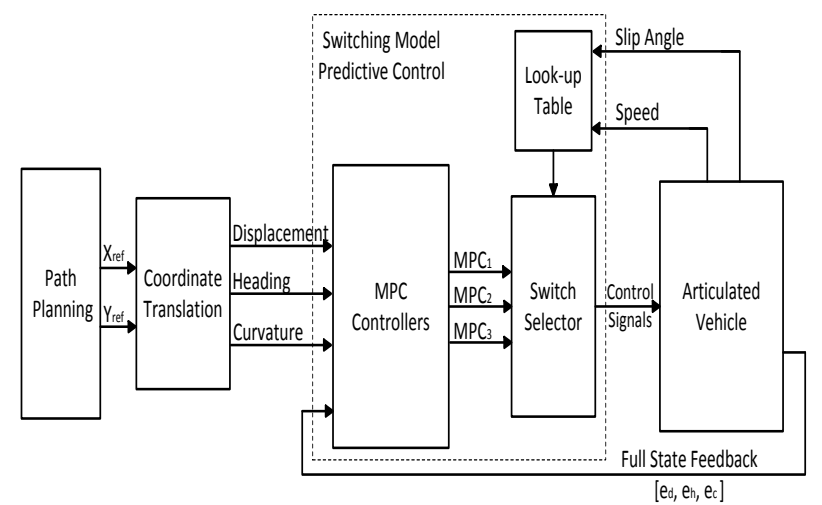

Fig 4: Switching model predictive control scheme block diagram for an articulated vehicle

The construction of the MP-controller is based on the system description defined in the objective function equation. The mode selector signal $i \in \mathscr{S}$ with $\mathscr{S} \triangleq\{1,2, \cdots, s\}$ is a finite set of indexes and $s$ denotes the number of switching sub-systems.

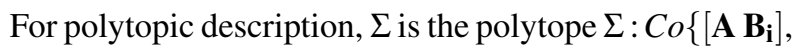
$\left.\cdots,\left[\begin{array}{ll}\mathbf{A} & \mathbf{B}_{\mathbf{s}}\end{array}\right]\right\}$, Co denotes the convex hull and $\left[\mathbf{A}, \mathbf{B}_{\mathbf{i}}\right]$ are the vertices of the convex hull. Any $\left[\mathbf{A}, \mathbf{B}_{\mathbf{i}}\right]$ within the convex set $\Sigma$ is a linear combination of the vertices $\sum_{j=1}^{s} \mu_{j}\left[\begin{array}{l}\mathbf{A} \\ \mathbf{B}_{\mathbf{i}}\end{array}\right]$ with $\sum_{j=1}^{s} \mu_{j}=1,0 \leq \mu_{j} \leq 1$.

In the presented methodology, the mode selector signal of the switching MPC is the measured slip angle for the front part of the vehicle $\beta$, while for defining the switching instances, the sets $\mathscr{L}_{i}$, will be discretized into equal operating subspaces. The discretization of the slip angles operating set, can be formulated by defining multiple nominal values $\alpha_{0}, \beta_{0}$ and allowing them to take values into neighbouring regions of lengths $\xi_{i}$ and $\psi_{i}$. This can be formulated as:

$$
\begin{aligned}
& \mathscr{L}_{1, i}=\alpha_{0, i}^{\min }=\alpha_{0, i}-\xi_{i} \leq \alpha_{0, i} \leq \alpha_{0, i}^{\max }+\xi_{i}=\alpha_{0, i}^{\max } \\
& \mathscr{L}_{2, i}=\beta_{0, i}^{\min }=\beta_{0, i}-\psi_{i} \leq \beta_{0, i} \leq \beta_{0, i}^{\max }+\psi_{i}=\beta_{0, i}^{\max }
\end{aligned}
$$

The sets $\mathscr{X}$ and $\mathscr{U}$ specify state and input constraints. Let the set $\mathscr{X}$ contain the $\mathbf{x}$ states that satisfy the following bounding inequality:

$$
\mathbf{x}^{\min }=\mathbf{x}-\Delta_{1} \leq \mathbf{x} \leq \mathbf{x}+\Delta_{1}=\mathbf{x}^{\max }
$$

where $\Delta_{1} \in \mathfrak{R}_{+}^{(3,1)}$ is the vector containing the selecting state boundary conditions. The control input bounding set $\mathscr{U}$ can be derived by taking under consideration the mechanical and the physical constraints of the articulated vehicle, as also the preference on aggressive or not maneuvers, these constraints can be also formulated as:

$$
u^{\min }=u-\Delta_{2} \leq u \leq u+\Delta_{2}=u^{\max }
$$

where $\Delta_{2} \in \mathfrak{R}_{+}$is the vector containing the selecting control boundary conditions. Let the matrix $\mathbf{H}_{\mathbf{i}}$ be a zeroed $2 \times 2$ matrix with its $i-$ th column equal to $[1,-1]^{T}$, and the other $\mathbf{0}_{2,1}$ i.e. for $i=2$ :

$$
\mathbf{H}_{\mathbf{i}}=\left[\begin{array}{cc}
0 & 1 \\
0 & -1
\end{array}\right]
$$

Then the previous bounds can be cast in a more compact form as:

$$
\left[\begin{array}{l}
\mathbf{H}_{1} \\
\mathbf{H}_{2}
\end{array}\right]_{4 \times 2} \cdot\left[\begin{array}{c}
\mathbf{x} \\
-- \\
u
\end{array}\right]_{2 \times 1} \leq\left[\begin{array}{c}
\mathbf{x}^{\max }+\Delta_{1} \\
----- \\
u^{\max }+\Delta_{2}
\end{array}\right]_{4 \times 1}
$$

These constraints are embedded in the MP-controller computation algorithm in order to compute an optimal controller that counts for the physical and mechanical constraints that restrict the articulated vehicle's motion. More specifically, the $(i)$-th MP-controller's objective is to optimize the quadratic cost in (14), while the $(i)$-th linearized system is within $\Sigma$.

Each model predictive controller $V_{M P C}^{i}$ corresponds to the $i$-th error dynamic model of the articulated vehicle, obtained by solving the following optimization problem $\min J(k)$, with respect to the control moves variations $\Delta u$ and the error coordinates, where $k$ is the discrete time sample index and $J(k)$ is the objective function to be minimized, which can be stated as a quadratic function of the states and control inputs:

$$
\begin{array}{r}
J(k)=\sum_{n=N_{W}}^{N_{p}}[\hat{\mathbf{y}}(k+n \mid k)-\mathbf{r}(k+n \mid k)]^{T} \mathbf{Q}[\hat{\mathbf{y}}(k+n \mid k)-\mathbf{r}(k+n \mid k)]+ \\
+\sum_{n=0}^{N_{c}-1}\left[\Delta u^{T}(k+n \mid k) \mathbf{R} \Delta u(k+n \mid k)\right] \\
+\sum_{n=N_{W}}^{N_{p}}[u(k+n \mid k)-\mathbf{s}(k+n \mid k)]^{T} \cdot \mathbf{N}[u(k+n \mid k)-\mathbf{s}(k+n \mid k)]
\end{array}
$$

where, $n$ is the index along the prediction horizon, $N_{w}$ is the beginning of the prediction horizon, $\mathbf{Q} \in \mathfrak{R}^{n} \times \mathfrak{R}^{n}$ is 
the output error weight matrix, $\mathbf{R} \in \mathfrak{R}^{m} \times \mathfrak{R}^{m}$ is the rate of change in control weight matrix, with $Q \geq 0$ and $R \geq 0$, $\mathbf{N}$ is the control action error weight matrix, where: $\hat{\mathbf{y}}(k+$ $n \mid k)$ is the predicted system's output at time $k+n$, given all measurements up to including those at time $k, \mathbf{r}(k+$ $n \mid k)$ is the output set-point profile at time $k+n$, given all measurements up to including those at time $k, \Delta u(k+n \mid k)$ is the predicted rate of change in control action at time $k+n$, given all measurements up to including those at time $k, u(k+n \mid k)$ is the predicted optimal control action at time $k+n$, given all measurements up to and including those at time $k$, and $\mathbf{s}(k+n \mid k)$ is the input set-point profile at time $k+n$, given all measurements up to and including those at time $k$. Moreover, $N_{p}$ is the prediction and $N_{c}$ the control horizons respectively.

Once all $V_{M P C}^{i}(k)$ controllers are computed, as it has been indicated in Fig.4, the total switching MP controller is constructed by implementing a switching among the difference controllers in relation with the estimated or measured values of slip angles $\beta$ and $\alpha$. At this point it should be noted that this article will not focus on how the slip angles are estimated or measured but it will assume that during operation of the vehicle full measurement of the slipping angles can be obtained. Regarding the stability of the proposed switching control scheme, for the case of a linear and constrained system, as the articulated vehicle studied in this article, the stability of each separate MPC controller can be directly proven as it has been indicated in [25] (case 3.7.2.1). For the case of switching MPCs, even if the stability of the single MPC is guaranteed, no direct generalisation can be made for the overall closed loop system [26]. Such kind of stability investigation is a research topic very interesting but is should not be further investigated in this article since is is out of the scope of our contribution.

\section{SIMULATION RESULTS}

The proposed switching modeling and control scheme will be performed with the following vehicle's indicative characteristics: $l_{f}=0.6 m, l_{r}=0.8 m$, and a constant velocity of $u=2 \mathrm{~m} / \mathrm{sec}$, without a loss of generality and a direct expendability and applicability of the presented simulated results in different types of articulated vehicles. Moreover, a comparison will be performed among the cases where a single or multiple MP-controllers have been utilized to control the performance of the vehicle operating in an terrain under the existence of varying slip angles.

In the simulated case, the constraints for the MPC scheme have been imposed as it follows: the bounds on the articulated angle have been defined as $-0.785 \leq \gamma \leq 0.785 \mathrm{rad}$, and the bounds for the displacement error have been set at $-0.2 \leq e_{d} \leq 0.2$, while the proposed controller has been evaluated in several reference paths and indicative real life scenarios.

The parameters for the MP-controllers for the state and control action weighings matrices have been tuned according to the changes of the state space models, and the different encountered slip angles. Moreover, for all the simulated test cases, the prediction horizon has been set equal to $N_{p}=10$ and the control horizon to $N_{c}=5$, with a control interval of $0.2 \mathrm{sec}$. In all the presented simulations, the velocity has been set constant.

The first comparison concerns the demonstration of the slip angles and the effect in deteriorating the overall tracking closed loop performance of the articulated vehicle. In this case two simulated scenarios have been considered, where a fixed MPC controller has been utilized to control the vehicle by utilising the error dynamics model, with and without taking under consideration the effect of the slip angles. In Fig.5 the responses of the MPC control during tracking of a circular path are being displayed for the front and the real parts of the articulated vehicle in the non-slip case.

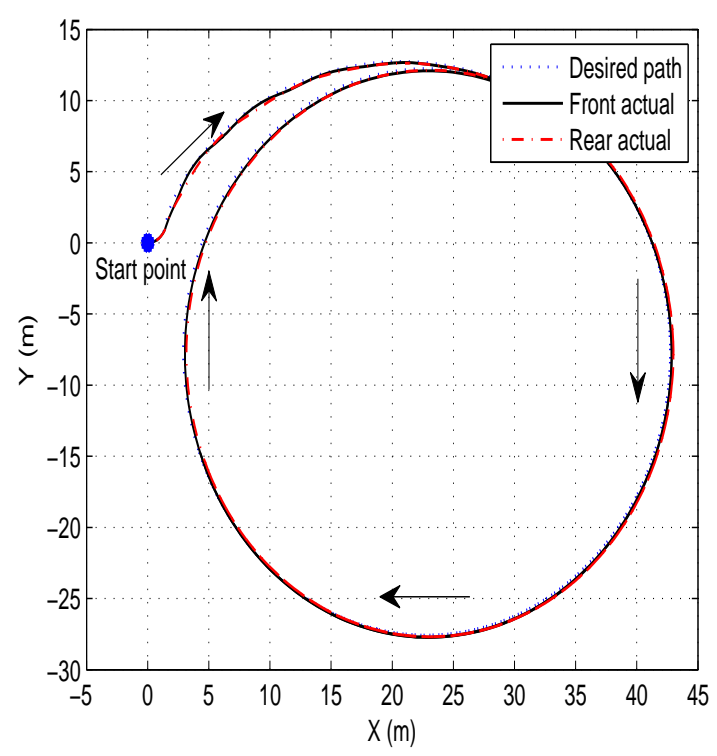

Fig 5: Single-MPC based circular path tracking for an articulated vehicle without considering the effect of slip angles

As it can be observed, the obtained responses are very smooth and with a very good tracking of the reference path, which leads to a zero tracking error. In the case that the error dynamics model, under the following existing slip angles, is being utilized with: $0 \leq \beta \leq 0.1 \mathrm{rad}$ and $0 \leq \alpha \leq 0.3 \mathrm{rad}$ and with the same fixed MPC controller, Fig. 6 displays the corresponding results.

In this simulated case it can be observed that the existence of the slippage deteriorates the overall tracking performance of the vehicle, while the MPC controller does not manage to provide a perfect path tracking behaviour for the articulated vehicle. Moreover, the effect of the slip 


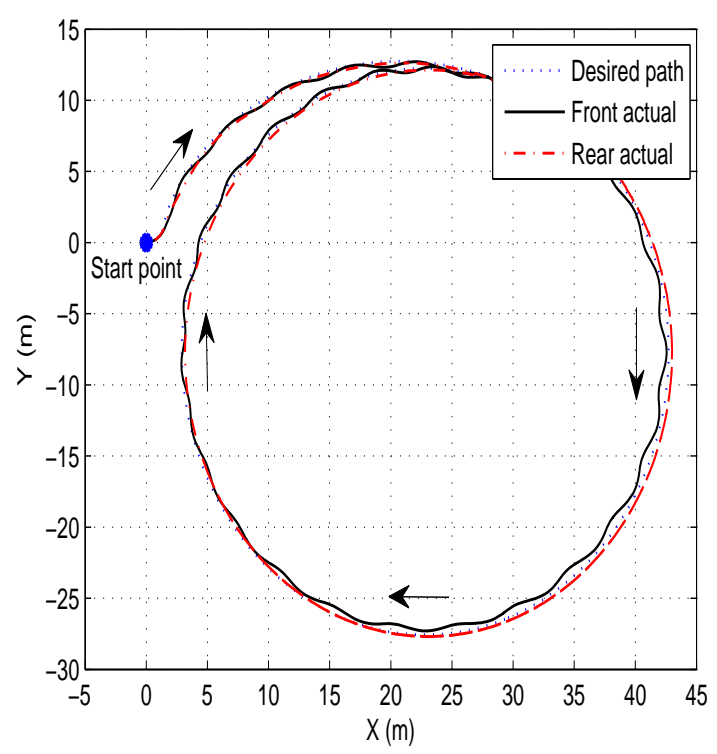

Fig 6: Single-MPC based circular path tracking for an articulated vehicle by considering the effect of slip angles

angles is more intense in the rear part of the vehicle and less in the front part.

Finally, in Fig.7 the fixed MPC control efforts needed for the non-slip and the slip case are depicted. Again it is obvious that the non-slip case contains more smooth control signals, since this case is ideal and less complicated than the slip case, where more intense control efforts should be applied to compensate the effect of slipping.

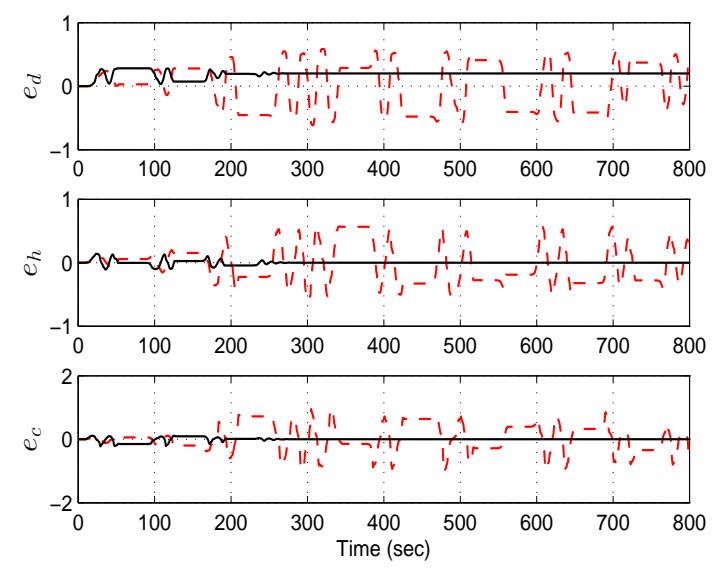

Fig 7: Single-MPC control efforts for the circular path under (red) and without (black) the influence of slip angles respectively

At this point it should be highlighted that this simulated test case is directly connected to the real life applications, since the slippage is existing and dramatically affects the behaviours of the vehicle. Based on the presented switching modeling and control approach established in this article, in the sequel the effect and the performance improvement that could be achieved, by applying the suggested scheme, under varying slip angles will be presented. The cases that have been considered are the ones defined in (Table 1), while for each case a MPC has been tuned. The selected deviations for $\alpha$ and $\beta$ have been inspired by a typical real life scenario slippage conditions and based on the analysis in $[13,25]$, while the different parameter tuning for the utilized MPCs are being presented in Table 2.

Table 1: Varying slip angles scenarios under constant ve-

\begin{tabular}{|c|c|l|}
\multicolumn{1}{|c|}{ locity } \\
\hline$i$ - MPC & $\alpha$ (degree) & $\beta$ (degree) \\
\hline 1 & $0 \leq \alpha<8$ & $0 \leq \beta<4$ \\
\hline 2 & $8 \leq \alpha<16$ & $4 \leq \beta<8$ \\
\hline 3 & $16 \leq \alpha<24$ & $8 \leq \beta<12$ \\
\hline
\end{tabular}

Table 2: Tuning parameters for the $i$-MPC

\begin{tabular}{|c|c|c|c|c|}
\hline$i$ & $Q$ & $R$ & $N_{c}$ & $N_{p}$ \\
\hline 1 & $100 \cdot \mathbf{I}$ & 0.1 & 5 & 10 \\
\hline 2 & $10 \cdot \mathbf{I}$ & 1 & 4 & 8 \\
\hline 3 & $\mathbf{I}$ & 10 & 3 & 6 \\
\hline
\end{tabular}

More analytically, in Fig.8, it is clearly presented that the proposed switching MPC has the merit of adapting to the varying articulated reference angle and achieve a good path tracking performance. Furthermore it should be highlighted that the utilization of only one fixed MP-controller fails to track the circular reference path. In this simulation result, the fixed $3^{\text {rd }} \mathrm{MP}-$ controller has been utilized, as this is the controller that had the best simulation output under constant velocity and varying slip angles, while the utilization of different fixed type controllers has resulted in a system instability. The presented results indicate the movement of the front part of the vehicle but similar results can be extracted for the real part.

In Fig.9 the corresponding time evolution of the vehicle's error dynamics $\left(e_{d}, e_{h}\right.$ and $\left.e_{c}\right)$, from the path tracking response in Fig. 8 are being displayed under a switching (black) and non-switching (red) MPC scheme. Finally, in Figure 10, the corresponding responses for the simulated time varying slip angles, for a path tracking response in Fig. 8 has also been presented, with respect to the switching mode selector, and control steering angle. Three operation sets for the slip angles have been defined from the simulation results. The total switching MPC is constructed by implementing a switching between the three controllers, indicated by the "SW" signal in Figure 10, 


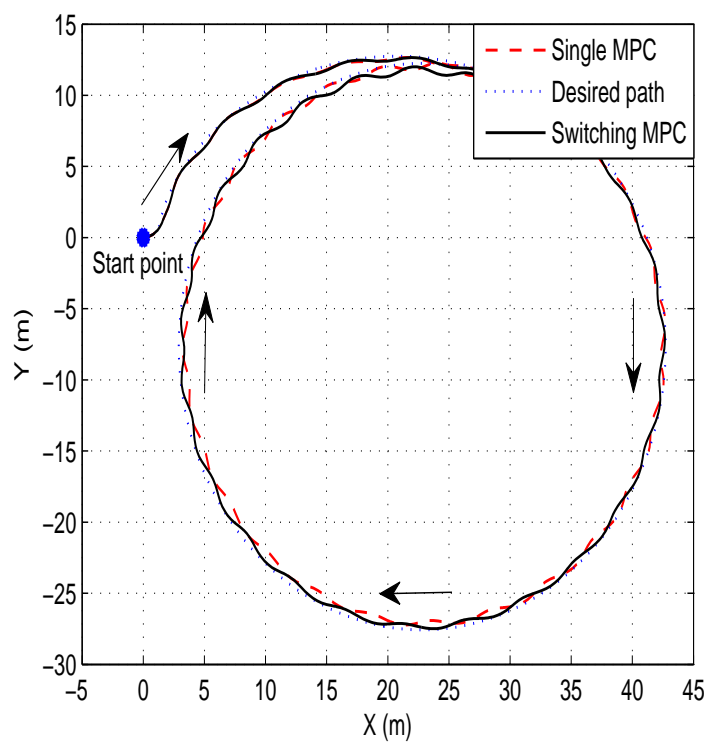

Fig 8: Circle path following based on switching-MPC (black) and single-MPC (red) under slip angles effects

with respect to the current values of the varying slip angles.
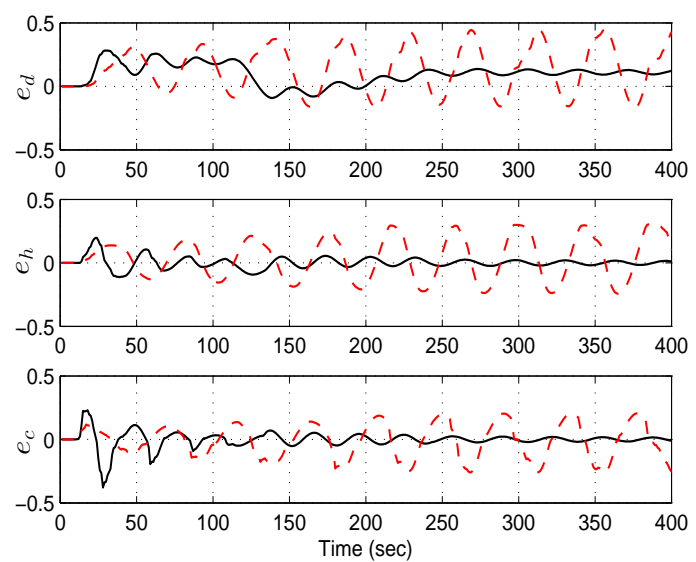

Fig 9: Time evolution of the system error dynamics for both case studied.

For evaluating the positioning errors among the actual path of the vehicle's wagons and the desired one, the path tracking errors have been utilized, that are calculated as the distance between the reference path and the position that the articulated vehicle experienced due to slip angles as presented in Fig.11. In this figure, the results from the error performance of the path tracking by utilizing switching and non-switching MPC under varying slip angles are being presented. As it can be observed there is a significant tracking error, due to the effect of slippage an-

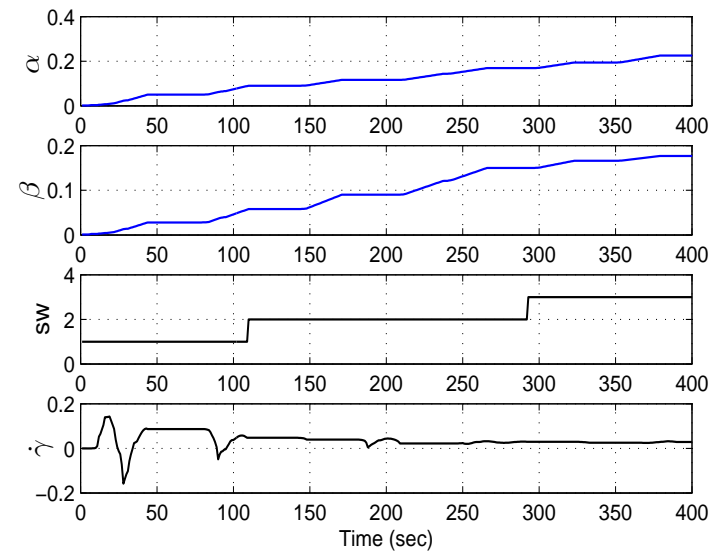

Fig 10: Time evolution of slip angles, switching mode selector and articulated angle as a control signal.

gels deteriorating the overall controller performance in the case of a single MPC. As it has been expected, again it is straight forward that the switching MPC scheme has a better performance than the fixed one.

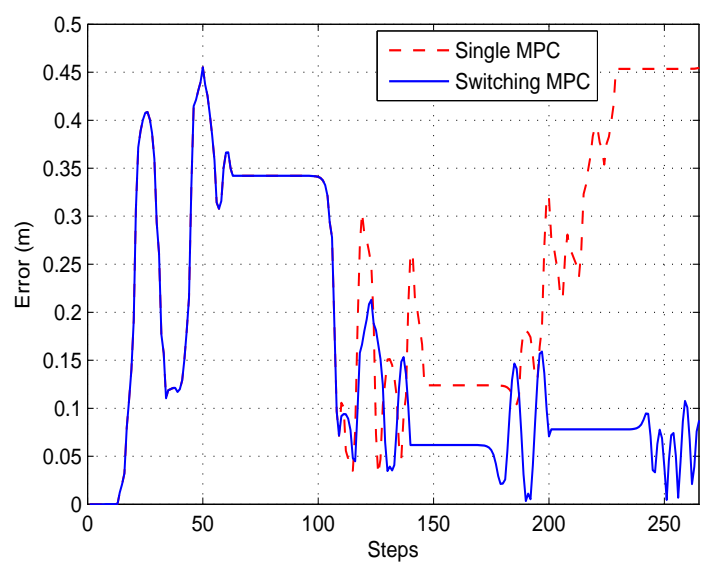

Fig 11: Tracking errors performance for the switching (blue) and the fixed (red) MPC schemes.

To extend the presented evaluation, the S-path curve has been utilized under the existence of varying slip angles. In Fig. 12 and Fig.13 the articulated vehicle's tracking response under the same controller configurations as in the previous case and the time responses of the system dynamics and the control signal are being displayed respectively. At this point it should be noted that the lower sub-figure of 13 presents a zoomed detail (first 100 samples) of the overall response for the $\dot{\gamma}$ to highlight the form and the time evolution of this signal. The time evolution of the error corresponding dynamics, $\left(e_{d}, e_{h}, e_{c}\right)$ from the path tracking response of the S-path, as in Fig.12 is being presented, where it can be also observed that there is a sig- 
nificant error in the state tracking in the case one utilized single-MPC.

As it can be observed from the obtained responses, the su-

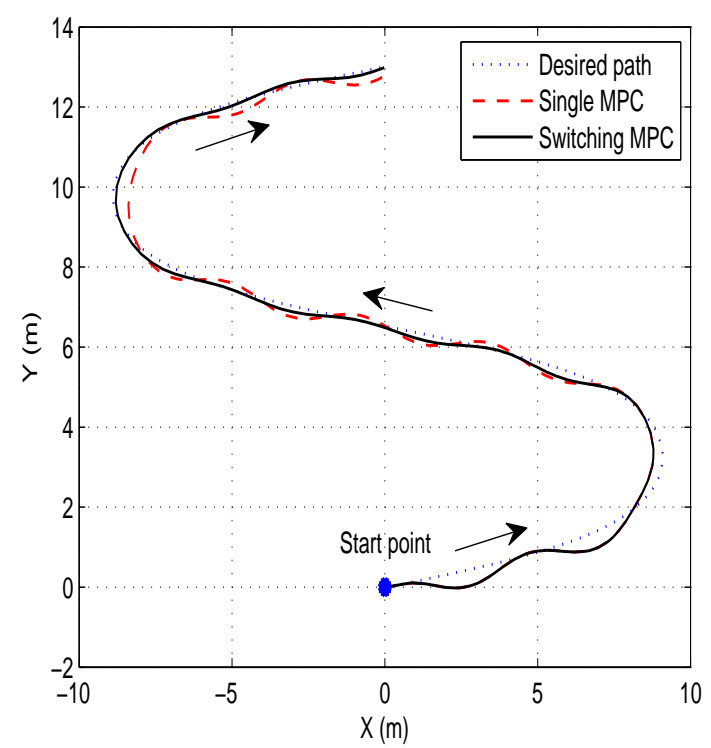

Fig 12: S-path following and switching (black) and fixed (red) MPC under varying slip angles.

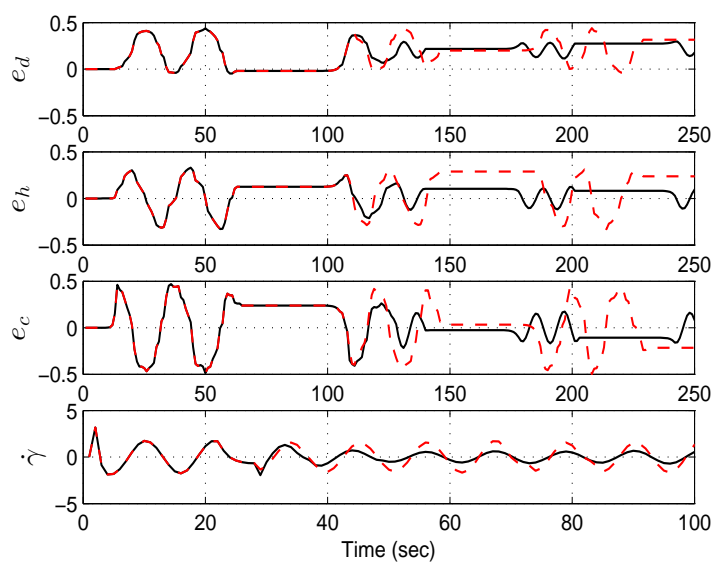

Fig 13: S-path following time evolution of error dynamics and corresponding control signal based on a switching (blue) and a fixed (red) MPC.

periority of the proposed switching modeling and control scheme for the articulated vehicle is straight forward. The switching scheme is able to provide an accurate and fast reference path tracking even in the case that we have sudden changes in the slip angles. To conclude the evaluation in this scenario, as in the previous case, the positioning errors among the actual path of the vehicle's wagons and the desired one are being presented in Fig.14. From this Fig- ure, it has ben clearly indicated that the proposed switching MPC has the merit of adapting to the varying slip angles, while being able to achieve a good path tracking performance. As it has been expected, again it is straight forward that the switching MPC scheme has a better performance than the fixed one.

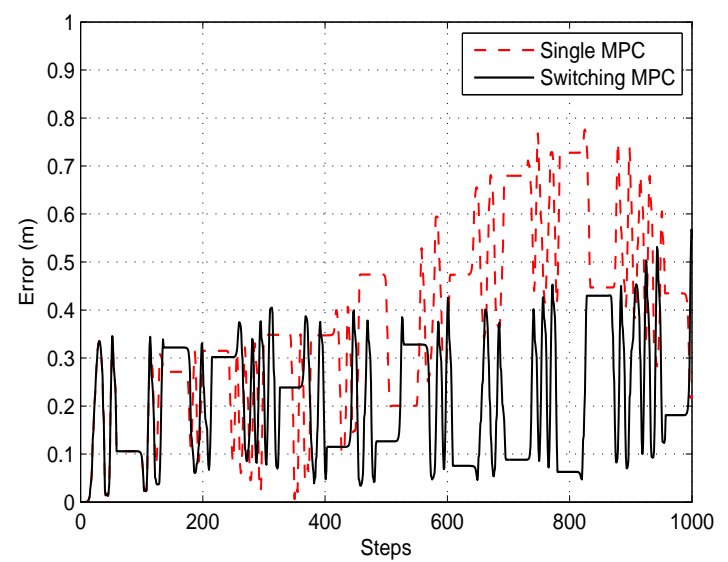

Fig 14: The error distance for the S-path.

Moreover, the proposed modeling procedure and switch MPC scheme, has been compared with the most commonly utilized control approaches in the field of articulated vehicles, which are the LQR and the Pole Placement (PP) control approaches. During this comparison, a circular path has been commanded, while for the switching MPC the previous presented tuning has been considered $(i=3)$. The settings for the calculated LQR gain were $Q=10 \cdot \mathbf{I}, R=4$, while the selected poles for the PP have been $[-0.8,-0.9,-1.9]^{T}$. In Figure 15 the obtained path tracking results are depicted, while Figure 16, the corresponding RMS path tracking errors have been also presented for a direct comparison. From the obtained results it is obvious that the proposed switching MPC is superior to the LQR and PP scheme, while it is able to track accurately the desired path.

For a final evaluation scenario, a realistic path, which is usually find in mining applications will be utilized, as the one presented in Fig.17, where additional boundaries (walls) have been included in the simulated scenario. The reference path for this case (blue) line has been considered as provided by a path planning algorithm.

As in the previous cases, the superiority and the capability of the switching MPC proposed scheme is obvious. The controller achieves a good tracking behaviour, which is necessary for tracking paths with an increased accuracy. The non existing overshoots and deviations from the desired path, makes the proposed scheme an ideal one, especially for real life applications and operations that needs to be performed in areas with constraints as the presented one. 


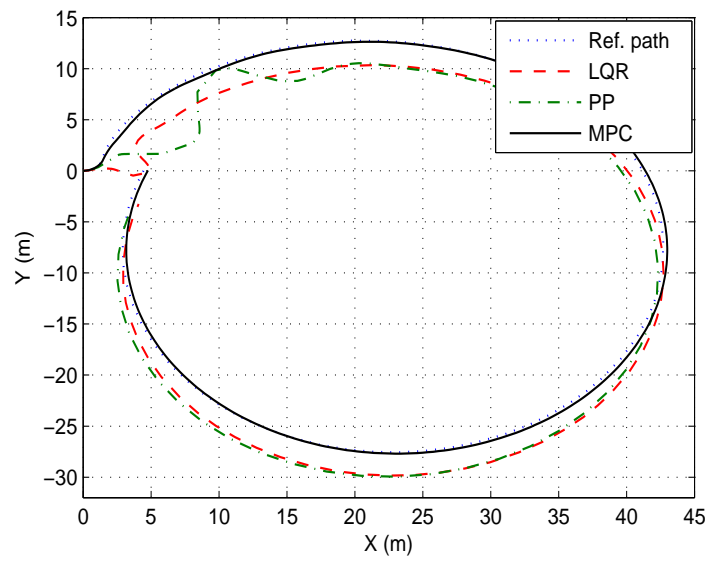

Fig 15: Tracking Performance comparison for a circular path based on switching MPC, LQR and PP under zero slip angles

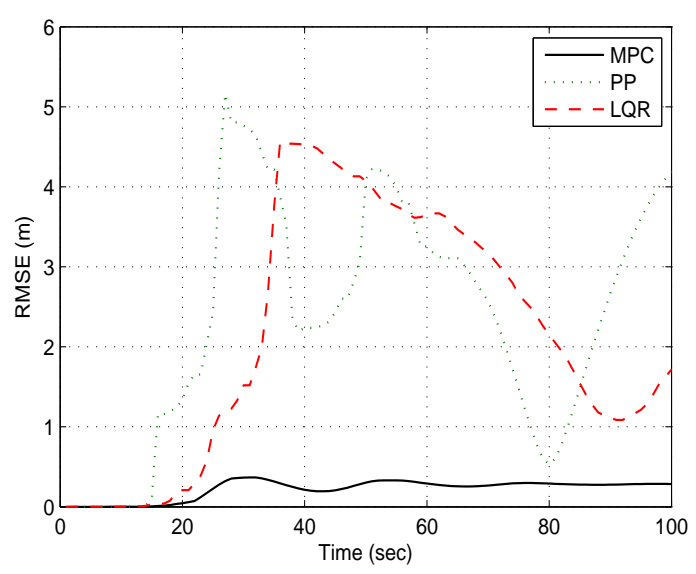

Fig 16: RMS Tracking errors comparison for a circular path based on switching MPC, LQR and PP under zero slip angles

As it has been presented until now, the effect of the slip angles on the overall performance of the vehicle is significant. In general, this effect can be expressed as a normalized Positioning Error Ratio (PER), which is the ratio of the slip radius against the ideal radius and can be defined as:

$$
P E R_{\theta}=1-\frac{\dot{\theta}_{s}}{\dot{\theta}}
$$

where the subscript $s$ denote the existence of slippage. As an example, the PER for the circular examined path and for the turning angle of the vehicle's front part is being presented in Fig.18, where the normalized positioning error ratio behaviour of the slip angles and the corresponding produced articulated angle are being displayed.

From a real life implementation of the switching MPC

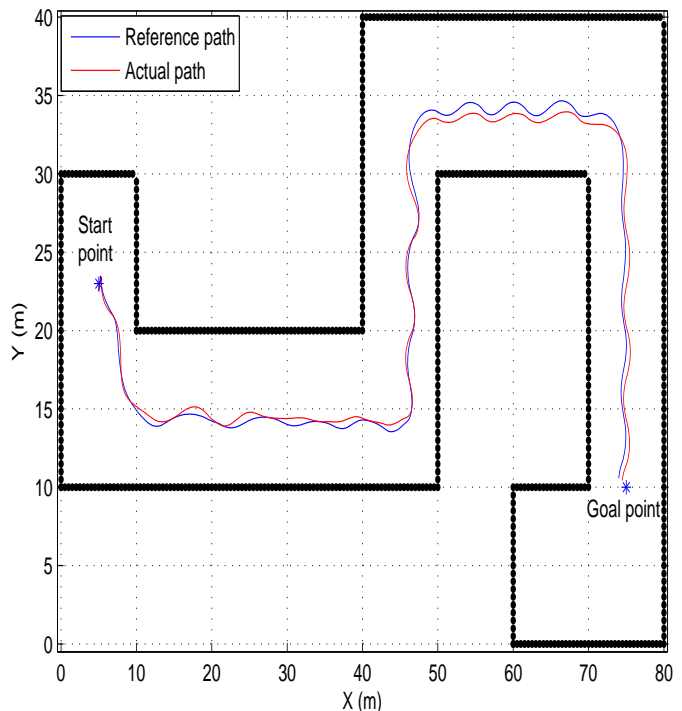

Fig 17: Articulated vehicle following a path in an arena having boundaries on both sides (reference simulated paths have been indicated by blue and red lines respectively

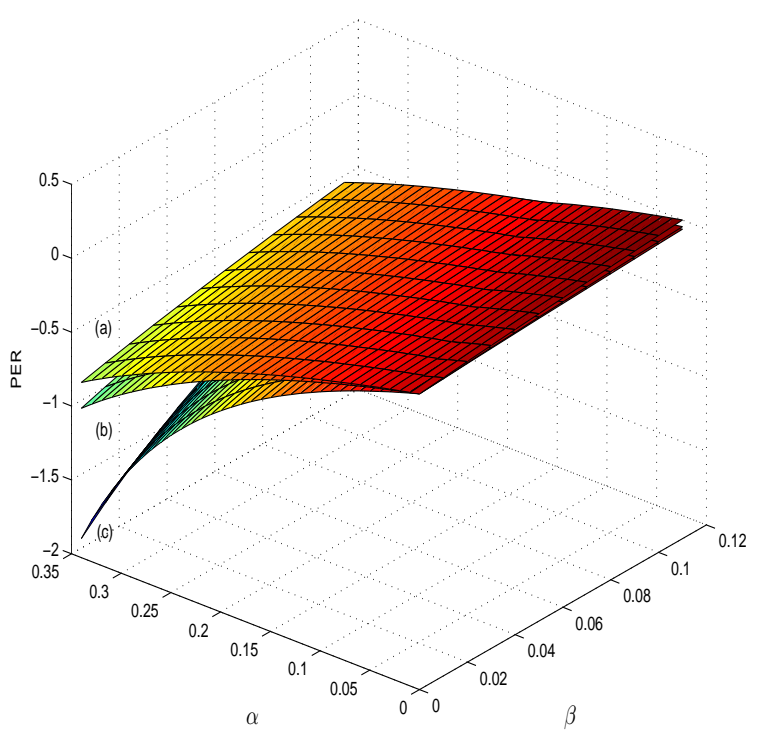

Fig 18: Positioning Error Ratio (PER) for slipping and non slipping comparison with different articulated angle scenarios; (a) $\gamma=0.523$, (b) $\gamma=0.698$, and (c) $\gamma=0.785 \mathrm{rad}$ 
scheme presented, it should be emphasised that the computational burden of the proposed methodology is being depended mainly by the selection of the ad hoc tuned prediction and control horizon $N_{p}, N_{c}$ parameters. This selection might not have been the optimal one, however based on numerous tuning efforts perfumed in this article and as it has been reported in [27], the computational complexity is monotonically increasing with respect to the increase of the $N_{p}$ and $N_{c}$ parameters, while in general smaller values are generally preferable. In Table 3 the computational burden is further analysed where the actual latency times, due to the MPC calculations are being presented, based on the same set of $Q=100 \mathrm{I}$ and $R=0.1$.

Table 3: Prediction Horizons for the $i$-MPC and related complexity in terms of computational latency times

\begin{tabular}{|c|c|c|}
\hline$N_{c}$ & $N_{p}$ & Actual time loop $(\mathrm{sec})$ \\
\hline 5 & 10 & 3.02405 \\
\hline 4 & 8 & 2.31053 \\
\hline 3 & 6 & 1.74104 \\
\hline
\end{tabular}

These results have been obtained based on a PC with a 2.67 GHz Processing Unit and 4 GB RAM and with MATLAB R2014a. As it can be observed, an increase in the prediction horizons directly affects the complexity, translated in actual loop latency time. However, it should be noted that in the exhaustive simulations, for $N_{c} \geq 3$ and $N_{p} \geq 6$ no significant performance improvement of the overall closed loop system has been achieved.

\section{CONCLUSIONS}

In this article a complete analysis towards the development of a switching modeling and control framework for an articulated vehicle, under the effect of varying slip angles has been presented. As it has been indicated by the multiple simulated test cases the established nonlinear kinematic model, of the nonholonomic articulated vehicle in cooperation with the proposed model predictive scheme are sufficient to provide a very good path tracking performance for the articulated vehicle, even under the case of varying slip angles that can significantly deteriorate the performance of the movement. The proposed scheme should be further utilized and applied in experimental studies to prove the overall efficiency and direct applicability of the suggested control scheme.

\section{REFERENCES}

[1] J. Markdahl, G. Bortolin, and U. Andersson. Traction control for articulated off-road vehicle. In Master Thesis, KTH Royal Institute of Technology, 2010.
[2] Yahja, Alex and Singh, Sanjiv and Stentz, Anthony. An efficient on-line path planner for outdoor mobile robots. Robotics and Autonomous systems, Elsevier, Vol. 32, No. 2 p.p. 129-143, 2000.

[3] C. Altafini. Why to use an articulated vehicle in underground mining operations? In International Conference on Robotics and Automation, IEEE, Detroit, Michigan, Vol. 4, p.p. 3020-3025, 1999.

[4] B.H. Pacejka. Tire and vehicle dynamics. (2nd ed.). Society of Automotive Engineers, Vol. 23, p.p. 147159, 2002.

[5] P. Petrov and P. Bigras. A practical approach to feedback path control for an articulated mining vehicle. In IEEE/RSJ, International Conference on Intelligent Robotics and Systems, Vol. 4, p.p. 2258-2263, 2001.

[6] U. Larsson, and G. Zell, and K. Hyyppa and A. Wernesson. Navigating an Articulated Vehicle and Reversing with a Trailer. IEEE, Proceedings, IEEE International Conference on, p.p. 2398-2404, 1994.

[7] Scheding, Steve and Dissanayake, Gamini and Nebot, E and Durrant-Whyte, H. Slip modelling and aided inertial navigation of an LHD. In Robotics and Automation, Proceedings, IEEE International Conference on, Vol. 3, p.p. 1904-1909, 1997.

[8] Bolzern, P. and DeSantis, R.M. and Locatelli, A. and Masciocchi, D. Path-tracking for articulated vehicles with off-axle hitching. In IEEE Control Systems Technology, Vol. 6, No. 4, p.p. 515-523, 1998.

[9] P. Bolzern and A. Locatelli. A comparative study of different solutions to the path-tracking problem for an articulated vehicle. In International Conference on Control Applications, Vol. 1, p.p. 427-434, 2002.

[10] D. Piyabongkarn, R. Rajamani, J. Grogg, and J. Lew, "Development and experimental evaluation of a slip angle estimator for vehicle stability control," IEEE Trans on Control System Technology, vol. 17, no. 1, pp. 78-88, January 2009.

[11] S. Scheding, G. issanayake, E. Nebot, and H. Durrant, "Slip Modeling and Aided Inertial Navigation of an LHD," in International conference on robotics and automation, New Mexico, 1997.

[12] F. Menn, P. Bidaud, and F. Amar, "Generic differential kinematic modeling of articulated multimonocycle mobile robots," Procceedings of the 2006 IEEE International Conference on Robotics and Automation, vol. 23, pp. 147-159, May 2006.

[13] S. Scheding, G. Dissanayake, E. Mario, and H. Durrant. An experiment in autonomous navigation of an underground mining vehicle. IEEE Trans. On Robotics and Automation, Vol. 15, No. 1, p.p. 8595, 1999.

[14] N. Hung, J. Im, S. Jeong, H. Kim and S. Kim. Design of a Sliding Mode Controller for an Automatic 
Guided Vehicle and Its Implementation. International Journal of Control, Automation, and Systems, Vol. 8, No. 1, p.p. 81-90, 2010.

[15] P. I. Corke and P. Ridley. Steering kinematics for a center articulated mobile robot. IEEE Trans. On Robotics and Automation, Vol. 17, No. 2, p.p. 215218, 2001.

[16] Astolfi A. and Bolzern, P. and Locatelli, A. Pathtracking of a tractor-trailer vehicle along rectilinear and circular paths: a Lyapunov-based approach. In IEEE Robotics and Automation, 2004.

[17] Tayebi, A. and Rachid, A. Path following control law for an industrial mobile robot. In Control Applications, Proceedings of the IEEE International Conference on, p.p. 703-707, 1996.

[18] Altafini, Claudio and Speranzon, Alberto and Wahlberg, Bo. A feedback control scheme for reversing a truck and trailer vehicle. IEEE Trans. on Robotics and Automation, Vol. 17, No. 6, p.p. 915922, 2001.

[19] P. Bigras, P. Petrov, and T. Wong. A LMI approach to feedback path control for an articulated mining vehicle. In 7th International Conference on Modeling and Simulation of Electric Machines, Converters and Systems, 2002.

[20] P. Ridley and P. Corke. Load haul dump vehicle kinematic and control. In Journal of dynamic systems, measurement, and control, Vol. 125, No. 1, p.p. 5459, 2003.

[21] Nayl, Thaker and Nikolakopoulos, George and Gustfsson, Thomas. Switching model predictive control for an articulated vehicle under varying slip angle. In Control \& Automation (MED), IEEE, 20th Mediterranean Conference on, p.p. 890-895, 2012.

[22] F. Nunez, S. Navarro, A. Aguado, and A. Cipriano. State estimation based model predictive control for LHD vehicle. In 17th World Congress, the International Federation of Automatic Control, 2008.

[23] F. Kuhne, J. Manoel, and W. F. Lages. Mobile robot trajectory tracking using model predictive control. In IEEE latin-american robotics symposium, 2005.

[24] Mayne, David Q and Rawlings, James B and Rao, Christopher V and Scokaert, Pierre OM, "Constrained model predictive control: Stability and optimality," Automatica, V. 36, No. 6, pp. 789-814, 2000.

[25] N. L. Azad, J. McPhee, and A. Khajepour, "Tire Forces and Moments and on-road Lateral Stabiliy of Articulated Steer Vehicles," SAE Technical Paper Series, No. 2005-01-3597, 2005.

[26] Liberzon, Daniel. Switching in systems and control. Springer, 2003.

[27] A. Bemporad, M. Morari, and N. L. Ricker, "Model Predictive Control Toolbox - User's Guide", Mathworks, 2014. 